\title{
Using Therbligs to embed intelligence in workpieces for digital assistive assembly
}

\author{
J. Oyekan ${ }^{1} \cdot$ W. Hutabarat ${ }^{1} \cdot$ C. Turner $^{2} \cdot$ C. Arnoult ${ }^{3} \cdot$ A. Tiwari ${ }^{1}$
}

Received: 6 July 2018 / Accepted: 9 April 2019 / Published online: 4 May 2019

(c) The Author(s) 2019

\begin{abstract}
Current OEM (Original Equipment Manufacturer) facilities tend to be highly integrated and are often situated on one site. While providing scale of production such centralisation may create barriers to the achievement of fully flexible, adaptable, and reconfigurable factories. The advent of Industry 4.0 opens up opportunities to address these barriers by decentralising information and decision-making in manufacturing systems through CPS (Cyber Physical Systems) use. This research presents a qualitative study that investigates the possibility of distributing information and decision-making logic into 'smart workpieces' which can actively participate in assembly operations. To validate the concept, a use-case demonstrator, corresponding to the assembly of a 'flat-pack' table, was explored. Assembly parts in the demonstrator, were equipped with computation, networking, and interaction capabilities. Ten participants were invited to evaluate the smart assembly method and compare its results to the traditional assembly method. The results showed that in its current configuration the smart assembly was slower. However, it made the assembly process more flexible, adaptable and reconfigurable.
\end{abstract}

Keywords Smart workpiece $\cdot$ Smart factory $\cdot$ Industry $4.0 \cdot$ Assembly operation

\section{Introduction}

Current OEM manufacturing facilities tend to be highly integrated and are often situated on one site. While providing scale of production, such centralisation may create barriers to the achievement of fully flexible, adaptable, and reconfigurable factories.

\section{Turner}

christopher.turner@surrey.ac.uk

J. Oyekan

j.oyekan@sheffield.ac.uk

W. Hutabarat

w.hutabarat@sheffield.ac.uk

C. Arnoult

c.arnoult@cranfield.ac.uk

A. Tiwari

a.tiwari@sheffield.ac.uk

1 Department of Automatic Control and Systems Engineering (ACSE), The University of Sheffield, Sheffield S1 3JD, UK

2 Rik Medlik Building, Surrey Business School, University of Surrey, Guildford, Surrey GU2 7XH, UK

3 Manufacturing Department, Manufacturing Informatics Centre, Cranfield University, Bedford, MK 43 0AL, UK
One particular vision for greater manufacturing flexibility, called Industry 4.0, has been put forward by the German government and national industries to envisage and promote the use of new technologies and organizational methods for manufacturing (German Federal Government 2016). Cyber Physical Systems (CPS) are a core theme of Industry 4.0 encompassing the further integration between machines and computing resources to form an intelligent network within the smart factory (Wang et al. 2015). The intelligence of the smart factory embodies four principles: (a) Interoperability between factory elements; (b) Ability to gather real-time information to enrich digital plant models; (c) Provision of manufacturing systems to support humans by providing reliable information or by conducting a range of tasks and (d) Promotion of distributed decision-making throughout factory elements allowing for, at present, partial machine autonomy.

However, smart systems and smart machines alone are not enough to achieve the goal of the Industry 4.0. In order to fully exploit the full potential of modern manufacturing technology, it is the opinion of the authors that workpieces must also be informated and become active intelligent components within the smart factory i.e. become smart workpieces. 
In this paper, a smart workpiece is defined as a part that, through the integration of additional electronic components, has the ability to communicate information to other workpieces, humans and production line machines/robots. Current research in the area of smart workpieces or parts focuses mainly on the use of embedded Radio Frequency Identification (RFID) tags to gather real-time data in order to improve the monitoring of production or scheduling/logistics/inventory processes (Zhong et al. 2013). In Velandia et al. (2016), the authors investigated how manufacturing, assembly and service data could be captured via RFID tags embedded in crankshafts and integrated with higher level business applications. However, in the case of RFID, the tagged workpieces are passive (Velandia et al. 2016).

In this work, we investigate the possibility for smart workpieces to actively participate in assembly operations by providing information to other workpieces and to the operator. The concept of smart workpieces communicating actively between each other and with the operator to perform assembly motion is a relatively new research area. In the next section pertinent works related to the research of this paper are discussed in more detail.

\subsection{Relevant research}

The continuing evolution of computing technology has significantly changed the face of manufacturing facilities and given rise to the most recent digital manufacturing paradigm of Industry 4.0. Computer Integrated Manufacturing (CIM) as term has been in existence since the 1970s. A definition for CIM can be found in Goldhar (1984) as 'a computer system in which the components are machine tools, robots and other processing equipment'. For Savage (1987) CIM refers to a management philosophy with the principle to realign the two most fundamental composite resources, people and machines. Thus CIM is not only the integration of mechanical, electrical or informational system it is a reflection of a new way to manage resources (Savage 1987). The definition given by the Computer and Automation System Association (CASA) of the Society of Manufacturing Engineers (SME) is often considered as the most comprehensive definition:

" $\mathrm{CIM}$ is the integration of the total manufacturing enterprise through the use of integrated systems and data communications coupled with new managerial philosophies that improve organizational and personnel efficiency." (Shrensker 1990).

Before the notion of the integrated enterprise different departments of a company used to be isolated from each other; often operating in separate functional silos each with its own proprietary data sets, methods, software and hardware to fulfil its role in the manufacturing business. A key theme of CIM has been to develop the notion of a shared computer data store to bring islands of enabling technologies

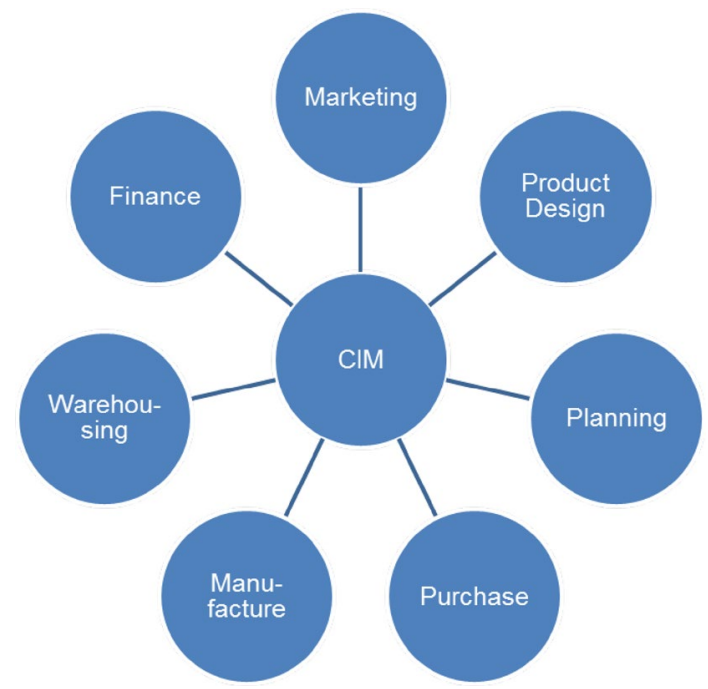

Fig. 1 Centralized CIM architecture

into a networked manufacturing system (Yu et al. 2015). Figure 1 illustrates this global and centralised architecture.

When CIM integration is successful the benefits in operational efficiency are clear, however there are many obstacles that can complicate the integration process. Integrating components provided by different suppliers can be particularly difficult and time-consuming. To achieve most integration projects a significant investment and substantial timescale required (Alavudeen and Venkateshwaran 2008). The integration of computer and advanced manufacturing technologies requires the creation of a structured, centralized and static business architecture ( $\mathrm{Yu}$ et al. 2015). Once this architecture is defined it is not particularly flexible. A significant investment in time is required to program the behaviour of a machine. However, the behaviour of a machine depends on the products it manipulates complicating the production of customised products (Alavudeen and Venkateshwaran 2008; Esmaeilian et al. 2016). In addition continuous maintenance and cleaning of the data that machines utilise is required (Alavudeen and Venkateshwaran 2008).

As it has been said previously, today's factories are facing new challenges on account of their integrated and centralised form, restricting flexibility in the production line. The vision of Industry 4.0 is focussed on the realisation of the "smart" factory that addresses such production concerns. The concept of Industry 4.0 has been inspired by the paradigm of holonic manufacturing.

The concept of holons was coined by Koestler (1968). In 1989 , as the CIM concept was considered unable to make production facilities adaptable, flexible and reconfigurable, the concept of holonic manufacturing was introduced (Suda 1989). 
The thematic research consortium HMS (Holonic Manufacturing System) of the International joint program IMS (Intelligent Manufacturing System) defined the principles of holonic application to manufacturing systems (Valckenaers et al. 1994):

Holon: An autonomous and co-operative building block of a manufacturing system for transforming, transporting, storing information and physical objects. The holon consists of an information processing part and often a physical processing part. A holon can be part of another holon.

In order to facilitate autonomy and co-operation capabilities a holon needs to be able to gather and analyse information. In effect holons need to be Cyber-Physical Systems (CPS). CPS are defined as physical and engineered systems whose operations are monitored, coordinated, controlled and integrated by computing and communication (Rajkumar et al. 2010). CPS are said to be key in the management of big data and to ensure interconnectivity in manufacturing facilities (Lee et al. 2015). Agent technologies in connection with the management of holonic manufacturing entities has been investigated by Wang and Haghighi (2016) who propose the use of such a software approach for the coordinated control of CPS systems within holonic manufacturing system.

Another important enabler for holonic manufacturing is the cloud manufacturing paradigm. Cloud Manufacturing consists of the use of the Internet of Things, the computing cloud, virtualisation and service-oriented technologies inside the factory (Wu et al. 2013). This paradigm transforms manufacturing resources and capabilities into services. These services are managed so that they are shared according to the need of the manufacturing environment (Tao et al. 2011). By giving holons access to these services, it increases their ability to make decisions and enables their distributed presence throughout the factory facilitating the development of production line flexibility and adaptability.

In implementing various forms of mass customization, automotive manufacturers have encountered problems with current automation techniques. Mercedes Benz have been replacing robots with workers on the production line for certain models, returning to manual installation, due to variety of customer specified options that were available for selection by customers and complications with the installation of the additional parts (Gibbs 2016). In a new approach Mercedes have been investigating the use of smaller lightweight robots to be used to assist workers rather than replace them (Gibbs 2016), an aim of CPS use espoused by many Industry 4.0 authors and an aim of the research in this paper.

In this paper the adjective "smart" is used to describe objects that have both autonomous and co-operative capabilities of a holon. In today's manufacturing environment, smart systems and smart machines do exist, though to achieve holonic manufacturing new ways of integrating intelligence into parts must be developed. Research exists concerning smart objects use for decision support within, for example, inventory strategy (Tsamis et al. 2015) and shop floor operations (Qu et al. 2013). However, these works only consider the intelligence of the object as a management tool. Currently workpieces are manipulated by human operators or by robotic arms and machines whose operations are governed by a central computerized intelligent system. In distributing the intelligence among workpieces, so that the human operator, the robotic arm or the machine is able to receive contextual information or instruction in real time from parts in the assembly process may allow for the realisation of efficient automated mass production of highly customized and personalised products. It is also possible that the addressing of such a research area may provide groundwork for the future achievement of autonomous or semi-autonomous production scenarios.

\subsection{Methodology}

The use of smart workpieces for assembly tasks is a very novel concept and as a result, few research outputs are currently available. By making use of an Ikea table assembly use case, this research aimed to establish how smart workpieces can actively participate in assembly operations and the benefits or drawbacks of using smart workpieces over traditional assembly process.

\subsection{Making smart workpieces through the use of Therbligs}

In order to make the workpieces smart, elemental assembly motions, called "Therbligs", were used. There are 18 "Therbligs" that can be used to describe any task (Ferguson 2000). The model of a Therblig is presented in Fig. 2. These motions capture human activities during an assembly task. To perform a Therblig, the operator observes the environment and obtains information from it. This is then used to inform their actions. As a result, each Therblig can be said to be made up of action and information. Currently, traditional assembly workpieces do not have any of these capabilities; they do not obtain or provide any information nor do they

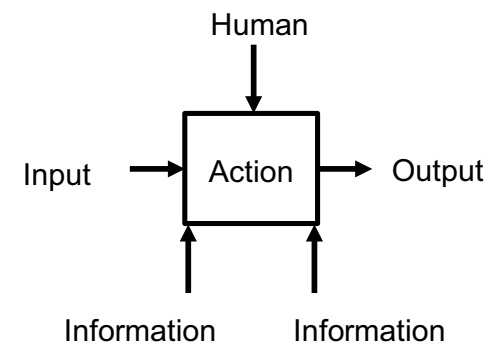

Fig. 2 IDEF0 model of a Therblig 
perform any action. In this context, they can be labeled as "passive" workpieces.

This research investigates the possibility of equipping a passive workpiece with the some capability of a human operator i.e. the information acquiring capability of a human operator, thereby turning it into a "smart" workpiece. Nevertheless, the current "smart" workpieces do not have the "action" capability and must rely on the actions performed human operators. As a result, in order for the smart workpiece to "perform" a motion, it communicates to the operator by signalling that an action must start. The operator performs the action until they receive another communication from the smart workpiece that the action must stop. As a result, the action transforms an initial state into the desired output state.

In Table 1, the motion column describes the Therbligs motions that a human carries out, the action column describes what the human typically does and the third and fourth column describes the information required or obtained at the start and end of an action that transforms a workpiece from an initial to a desired output state. Therbligs, such as "avoidable delay", "unavoidable delay" and "rest", have the action "waiting" or "resting". Thus, they can be said to be passive. These motions are consequences of human fatigue, human lack of control and/or overall factory problems. Nevertheless, by equipping smart workpieces with active motions in the future, such issues can be mitigated. In this work, we do not consider the Therbligs of avoidable delay and rest.

The Therbligs of "search", "find" and "plan" relate to human mental reaction and as the complexity of the assembly operation increases, the cognitive load on the operator increases. Smart workpieces could be used in order to reduce this cognitive load in the future. For example, the "find" phase and duration of the "search" operation can be reduced by making use of smart workpieces that provide information regarding their location to an operator. In order for this to happen, the information element has to be embedded into passive workpieces. As a result, the Therbligs of interest in this work are those with the information element emphasized. Consequently, the Therbligs of "search", "select" and "inspect" were chosen for embedding onto passive workpieces in order to make them smart.

\subsection{Use-case scenario development}

In order to investigate the possibility of making passive workpieces smart, the assembly of an IKEA table was used as a use-case scenario (shown in Fig. 3).

The assembly principle is presented in Fig. 4.

From Fig. 3 all four legs of the table are considered as different independent pieces. The idea was to create artificial complexity that will then be addressed through the use of smart workpieces. Creating artificial complexity is a method that has been investigated in works such as Prahbu et al. (2015). Artificial complexity was introduced by differentiating the legs of the table using patterns as shown in Fig. 5; and each pattern must be positioned at a particular corner of the table during assembly. Each corner was associated to a particular shape: triangle, square, pentagon and circle as shown in Table 2 and Fig. 8. Further artificial complexity was introduced by enforcing a certain sequence in which the legs needed to be assembled into the table corners however, the operator did not know the sequence (see Fig. 6) unless he/she looked at a manual which added more time to the assembly process. However, this could be solved by embedding information providing elements into the "passive" workpieces.

\subsection{Embedding information element into passive workpieces}

In order to make the workpieces smart they have been equipped with a low-cost, basic microcomputer called Raspberry Pi. The Raspberry Pi is used to control the electronic components that equip the workpieces (sensors and LEDs). They can also send to and request information from other workpieces so that they kept in sync in regards to the progress of the assembly task.

The electronic components used on the legs and the table top are different. On a leg, the microcomputer controls an LED (Light-Emitting Diode). This LED is set up to give a light signal that can be observed by the operator or read by the sensors situated on the table.

Figure 7 shows a smart leg developed as part of this research. The table top was equipped with four colour sensors TCS 34725 (TAOS 2016). These sensors were used to detect the light signals given by the smart legs. Each of them was placed in a corner and is able to recognize the particular light signal emitted by the appropriate leg (the one to position in this particular corner). Figure 8 represents the smart table top.

To launch the assembly process and receive data from the smart workpieces the operator uses a microcomputer. This computer is connected to a screen so that the information can be read by an operator. The smart workpieces need to communicate with other parts and also with the operator. Towards this, a Wi-Fi router was used to create a virtual private network composed of the smart workpieces' and operator's microcomputers. Within the network, each microcomputer has a fixed IP address so it is possible to identify each workpiece and the operator's computer.

In order to facilitate communication between workpieces, a client-server communication protocol was established. Data is published on a server and clients request access to the server so that they can collect the information they need. 
Table 1 Analysis of the Therbligs

Motion
Search: Begins when the eyes and/or the hand start to
seek the part and ends when the part is located
Find: Defines the momentary mental reaction at the end
of the Search cycle
Select: Choosing a particular object among a group of
similar object
Grasp: Starts when the active hand grabs the object and
ends when the next operation (use or transport loaded)
starts
Hold: The retention of a part after it has been grasped,
with no other movement or manipulation of this part
taking place. For example, one hand can hold the grasp
of a part while the other hand is performing the assem-
bly of another piece

Transport loaded: Moving a part using a hand motion

Transport Empty: Moving the unloaded hand

Position: placing the part in the proper orientation for performing the motion "use"

Assemble: joining two parts together

Use: manipulating an object in the way it is intended to be manipulated

Disassemble: separating parts that were joined

Inspect: comparing the object with a predetermined standard

Preposition: replacing an object in the proper orientation for its next "use". The positioning does not have to be precise as motion "position" is performed after

Release Load: releasing the object when it has reached is destination

Unavoidable delay: period from the point when a hand is inactive to the point when it becomes active again. These delays are out of the control of the worker (lack of raw materials, repair of a tool...) and might be dealt with the overall factory/business system

Avoidable delay: waiting within the worker's control which causes idleness that is not included in the regular work cycle

Plan: Mental function which may occur before "assemble" (deciding which part is going next) or prior to "inspect" noting which flaws to look for

Rest (to overcome the fatigue): A lack of motion which is only found when the rest is prescribed by the job or taken by the worker

$\begin{array}{lll}\text { Action } & \text { Information to start } & \text { Information to end } \\ \text { Looking around } & \text { "Identity" of the searched part } & \text { Location of the searched part } \\ \text { Looking around } & \begin{array}{c}\text { Recognisable characteristic of the } \\ \text { searched part }\end{array} & \begin{array}{c}\text { Matched characteristic of a } \\ \text { part in the search area } \\ \text { Location of the part that } \\ \text { matches the criteria }\end{array} \\ \text { Grasping } & \begin{array}{l}\text { Criteria of selection } \\ \text { Identity of the object to grasp }\end{array} & \begin{array}{c}\text { Confirmation that the object } \\ \text { is grasped }\end{array}\end{array}$

Holding Identity of the object to hold Order to hold

Order to do the next motion

Moving

Moving

Positioning

Assembling

Using

Disassembling

Inspecting

Prepositioning

Releasing

Waiting

Planning

Resting
Identity of the part to move

Location of the part

Destination of the part

Destination of the hand

Identity of the part to position Initial orientation of the part Desired orientation

Identities of the Parts to assemble The way to assemble them

Identity of the object to use The way to use it

Identities of the Parts to disassemble

The way to disassemble them

Identity of the object

Predetermined standard

Identity of the part to position Initial orientation of the part Desired orientation

object to release

Problem occurring: shortage, delay...

Problem occurring

Need for the worker to rest
Confirmation that the part has reached its destination

Confirmation that the hand has reached its destination

Confirmation that the part is oriented as desired

Confirmation that the parts are assembled

Confirmation that the use motion is finished

Confirmation that the parts are disassembled

Result of the comparison between the object and the standard

Confirmation that the part is oriented as desired

Confirmation that the object has been released

Information that the problem have been solved

Information that the problem have been solved

Structured sequence defining how things are going to happen

End of the resting period
In the use-case scenario two servers are present. The first one is located on the operator's computer and contains the state of the LED as well as the name of the active sensor on the table top. Its clients are the legs and table's microcomputer that request access to the server to read the data in order to activate the appropriate LEDs and sensor. The second server is located on the table and contains the results of the pieces' inspection. Its client is the operator's computer that 


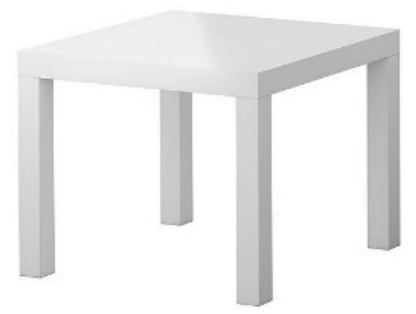

Fig. 3 The IKEA table

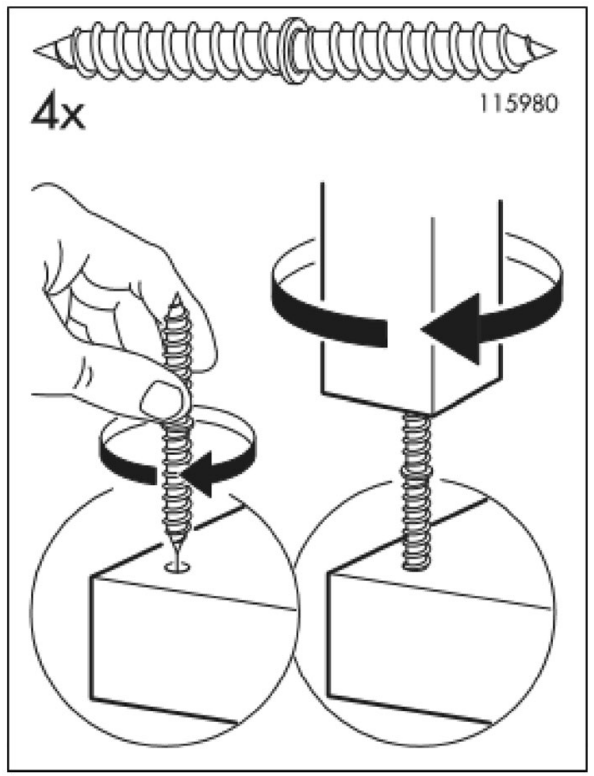

Fig. 4 The assembly principle of the table

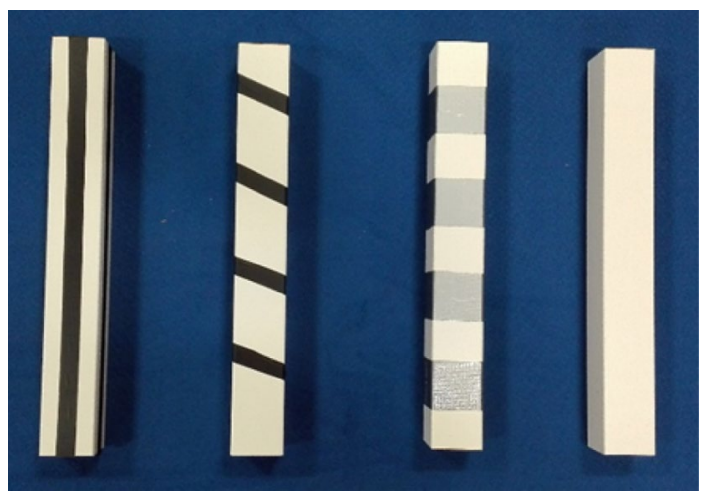

Fig. 5 The four legs with different patterns printed on them

requests data in order to display the result of the inspection on the operators screen. Workpieces need to continuously request access to the servers to see if the data has changed. If the data has not changed, the workpieces need to read it in order to know that no changes have happened. If the data
Table 2 Correspondence between legs and corners
The leg...
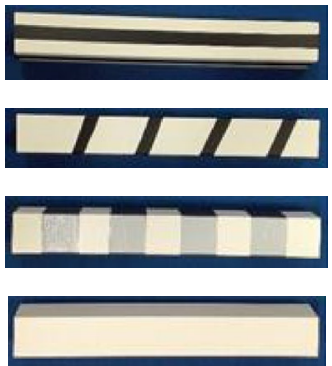

... must be assembled in the hole...

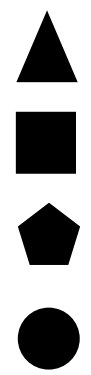

has changed the workpieces need to read it to be aware of the changes. Figure 9 illustrates the information flow in the smart object network.

\subsection{Implementation of smart "search/select"}

As can be seen in Fig. 6, the motion "search" appears twice in the assembly sequence of the scenario: searching for the leg; searching for the associated corner. In this work, the smart "search" was only implemented for the leg and not for the corner. However, a similar principle to the one used for implementing the smart "search" for the leg can be used to implement the corner smart "search". On the operator's computer, the program sets the state of the legs' LEDs to one of three states namely: "on", "off" or "blink". Initially, all the LED's states are "off" and only the active leg's LED's state is set to "on" by the program. The LED's states are updated in real time and published to a file. This file represents the part of a database available on the operator's computer server. As all the workpieces are part of the network, the leg based microcomputers are able to read in real time the information published in this database. They send a request to the server to obtain the state of the other LEDs and the server provides the requested data. Figure 10 shows the sequence diagram for 'start search'. All 'leg' computers read the state of their LED. If the state is "off", the LED is switched off. If the state is "blink", the LED starts to blink to a certain frequency. If it is "on", the LED switches on. The leg with the switched on LED is actually the active leg, The activity diagram of a LED is presented in Fig. 11.

This smart "search" implementation for the leg is actually reducing the cognitive load of the operator so that instead of remembering the patterns of the leg he/she must manipulate, the operators must simply find the leg on which the LED is switched on.

\subsection{Implementation of smart inspect}

The sequence diagram for the Inspect motion is presented in Fig. 12. When the inspection starts, the operator's computer 
Fig. 6 Sequence of assembly motions analysed using Therbligs. This sequence is repeated for each of the four legs. In this scenario, the motions "select" and "search" are similar because there is only one group of object (the legs) that are part of this experimentation

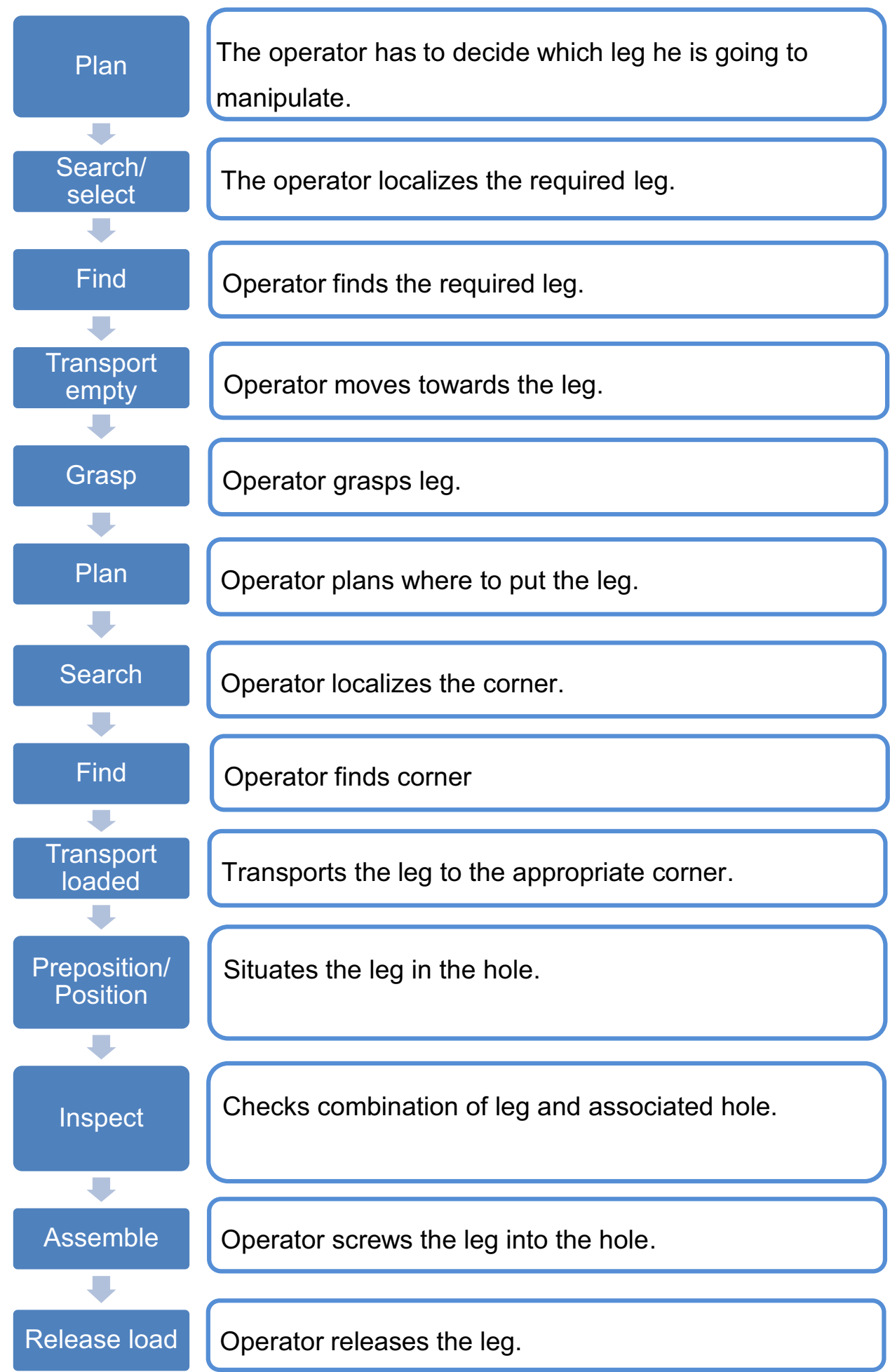

sets the active leg's state to "blink" in the database "stateComponents". Each leg has its own blinking frequency as seen in Table 3.

The operator's computer also sends the name of the active sensor to the database (one of four options: "triangle"; "square"; "pentagon" or "circle"). The active leg requests in real time the evolution of its state to the database. Since the active LED's state is "blink", the active LED starts to blink to its particular frequency. The table top's microcomputer requests in real time the name of the active colour sensor. Figure 13 shows the activity diagram for the colour sensors' activation. It can be noted that this diagram does not 


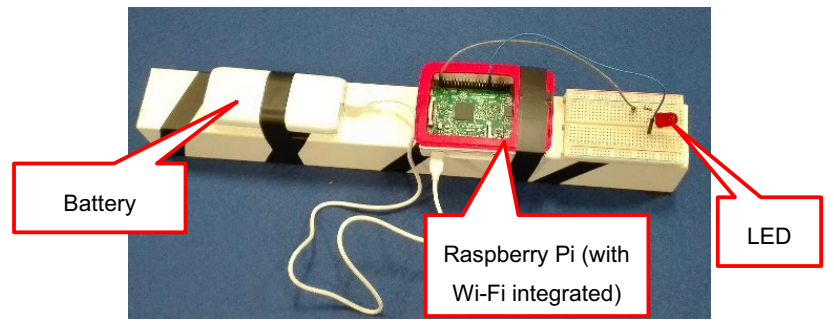

Fig. 7 A smart leg workpiece

present the deactivation of the sensors. The activate sensor is automatically deactivated if the right corresponding leg is placed in the corner. As the active leg has already been positioned facing the sensor (the operator must have followed the instruction "Turn the leg until its LED is facing the sensor and then press 'Enter" which was displayed on his screen), the active sensor on the table top can start reading the blinking frequency of the LED.

The active sensor sends in real time the result of its reading to the file that is representing a database available on the server. The activity diagram for the active sensor is presented in Fig. 14. If the blinking frequency of the LED on the leg corresponds to this corner (the corner in which the active sensor is positioned), then the state of the variable inspection is set to "good leg" in the database and the active sensor is deactivated. If the blinking frequency of the LED is the one of a leg corresponding to another corner, then the state of the variable inspection is set to "wrong leg" in the database. Otherwise, the state of the variable inspection is set to "no detection". In the last two cases, the active sensor is not deactivated so to enable the operator to repair his mistake by replacing the wrong leg with the correct one. The active sensor keeps reading the blinking frequency until it detects the expected leg.

\subsection{Experiment setup}

In order to test and validate the work, ten people were invited to complete two experiments. The first experiment consisted of the manual assembly of the IKEA table. To perform this task, the volunteers had at their disposal an instruction manual as well as a checklist sheet. After assembling a leg, the operator indicates on the sheet which leg they

Fig. 8 The smart table top

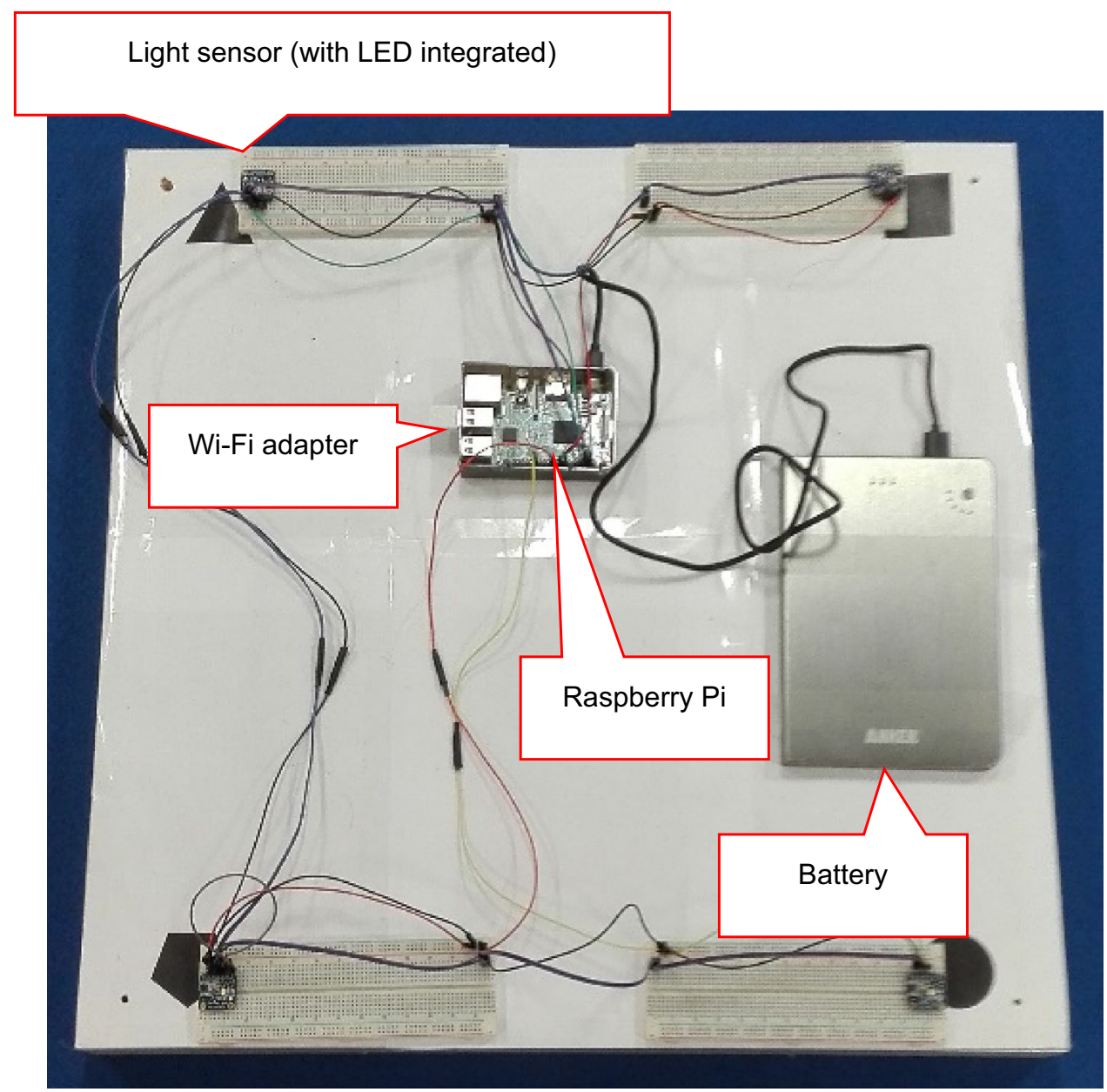


have associated with each shape. The second experiment consisted of the assembly of the table using the smart workpieces. The operator has at their disposal a computer running the "smart" program and a screen linked to the computer to display the information.

In order to make the two experiments (manual and smart) comparable, sequences of equivalent movements to be completed by the operator have been created for the two scenarios. The sequences for the assembly of one leg are presented in Table 4.

After completing the two experiments, discussion with the participants took place in which they gave their impressions and feedback of the process. They were then asked to fill a questionnaire to gather their opinions on the experiment. 10 participants were used in the experiments to compare manual assembly against smart assembly.

\section{Results of the experiments}

\subsection{Manual assembly}

Out of the ten participants, four said they were familiar with the concept of smart objects, two participants had heard of it and four did not know of the concept. As for the manual assembly scenario, seven of the volunteers found it intuitive. However, it was noted that although the manual indicates it, nine participants out of ten had spent a considerable amount of time to select the leg to manipulate. It was also found that 8 participants lost time checking leg to corner correspondence. The main problem encountered by participants was that they forgot to fill the checking sheet. It was not natural for them to make the check. One participant also mentioned that the process presented in this scenario was similar to flexible manufacturing where the same parts can go into an assembly at different times because of small differences between product variants.

\subsection{Smart assembly}

As for the smart assembly scenario, all the participants found it intuitive and they all thought that the instructions displayed on the screen were clear enough and easier than following a manual. In addition all participants found the smart assembly concept interesting. Nine participants would like to have this smart assembly guidance for every piece of self-assembly furniture. Eight of them would agree to pay a bit more for their furniture to have this smart assembly guidance.

Table 4: Summary of results from experiments. Where possible, we have used quantitative measures to show how the manual assembly process compares with the smart assembly process. The results seem to suggest that participants favoured the smart assembly process even though it took more time to use.

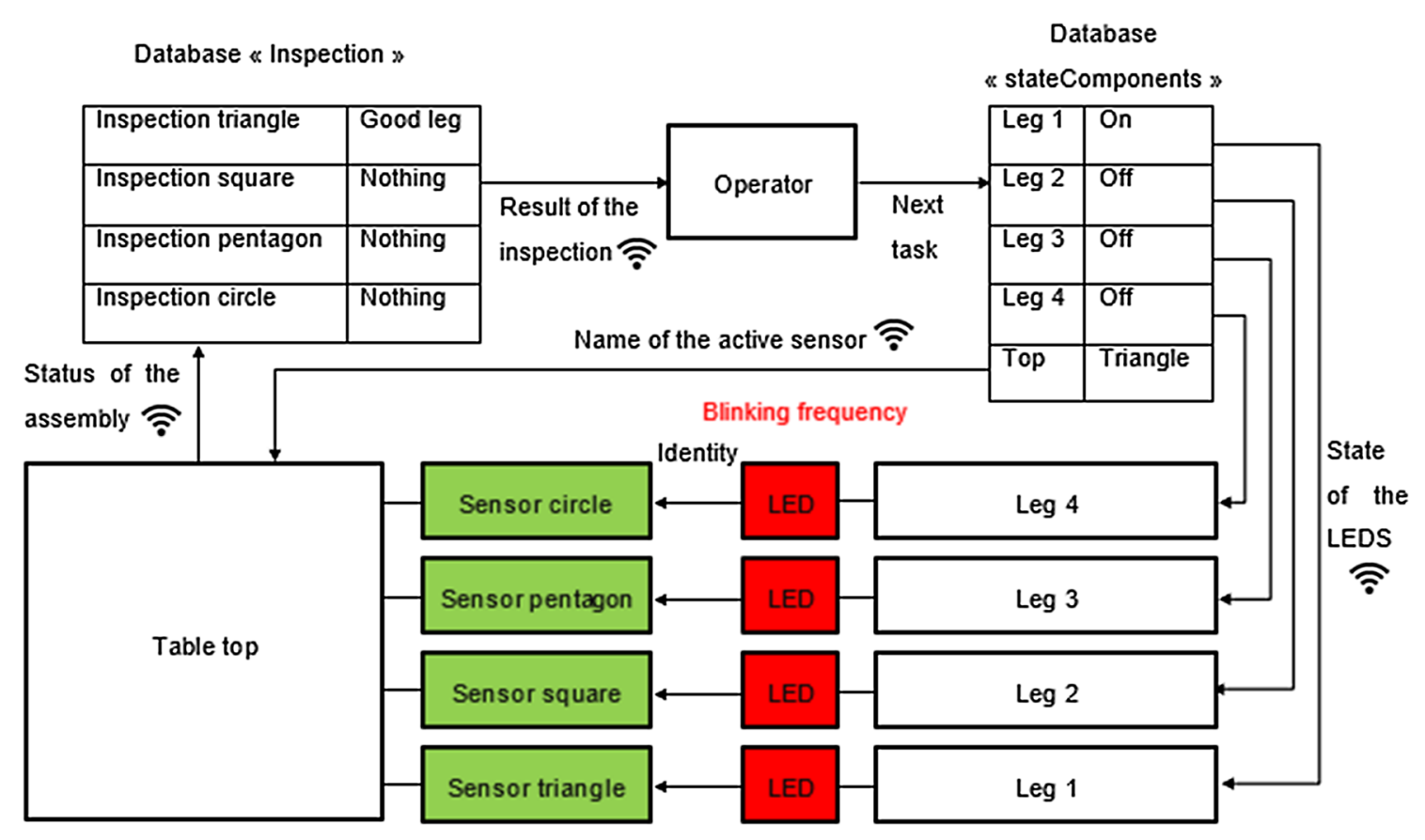

Fig. 9 Information flow diagram between workpieces and operator 


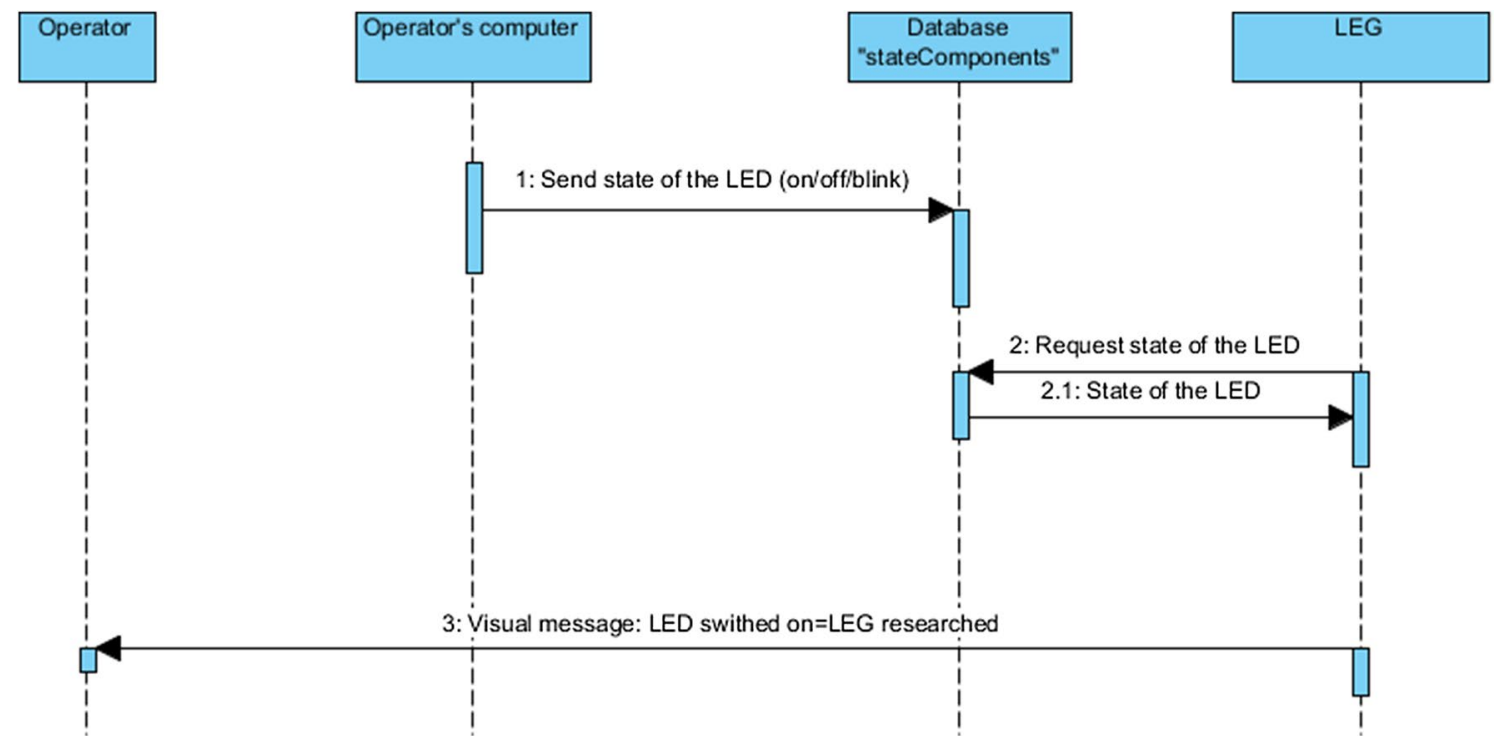

Fig. 10 Sequence diagram for smart search/select

\begin{tabular}{|c|c|c|c|c|c|c|}
\hline \multirow[t]{2}{*}{ Citeria } & \multicolumn{3}{|l|}{ Manual } & \multicolumn{3}{|l|}{ Smart } \\
\hline & Observations & Negative & Positive & Observations & Negative & Positive \\
\hline Was it intuitive to use? & $\begin{array}{l}\text { Seven of the volunteers } \\
\text { found it intuitive }\end{array}$ & 3 & 7 & All the participants found it intuitive & 0 & 10 \\
\hline $\begin{array}{l}\text { Difficulty selecting next } \\
\text { workpiece }\end{array}$ & $\begin{array}{l}\text { Nine participants out of ten } \\
\text { had spent a considerable } \\
\text { amount of time to select } \\
\text { the leg to manipulate }\end{array}$ & 9 & 1 & $\begin{array}{l}\text { They all thought that the instructions } \\
\text { displayed on the screen were clear } \\
\text { enough and easier than following a } \\
\text { manual }\end{array}$ & 0 & 10 \\
\hline $\begin{array}{l}\text { Time lost finding work- } \\
\text { pieces }\end{array}$ & $\begin{array}{l}\text { It was also found that } 8 \\
\text { participants lost time } \\
\text { checking leg to corner cor- } \\
\text { respondence }\end{array}$ & 8 & 2 & $\begin{array}{l}\text { They all thought that the instructions } \\
\text { displayed on the screen were clear } \\
\text { enough and easier than following a } \\
\text { manual }\end{array}$ & 0 & 10 \\
\hline Time used & $\begin{array}{l}\text { It took participants } 2 \text { min on } \\
\text { average to finish assembly }\end{array}$ & & & $\begin{array}{l}\text { It took participants } 2: 20 \text { min on average } \\
\text { to finish assembly }\end{array}$ & & \\
\hline $\begin{array}{l}\text { Will you pay extra for } \\
\text { smart work pieces? }\end{array}$ & & & & $\begin{array}{l}\text { Eight of them would agree to pay a bit } \\
\text { more for their furniture to have this } \\
\text { smart assembly guidance }\end{array}$ & 2 & 8 \\
\hline $\begin{array}{l}\text { How portable was the } \\
\text { smart workpiece? }\end{array}$ & & & & $\begin{array}{l}\text { The participants were asked what would } \\
\text { be the most important improvement } \\
\text { to be made to the physical design of } \\
\text { the workpieces and they answered } \\
\text { that the embedded equipment should } \\
\text { be smaller, lighter and integrated. In } \\
\text { particular the point was made that the } \\
\text { process was slower than it could be } \\
\text { because of size and weight problems }\end{array}$ & & \\
\hline $\begin{array}{l}\text { Documenting assem- } \\
\text { bly process (This is } \\
\text { needed in manual } \\
\text { assembly lines to tell } \\
\text { the next stage of the } \\
\text { process what has been } \\
\text { done in the previous } \\
\text { stage) }\end{array}$ & $\begin{array}{l}\text { The main problem encoun- } \\
\text { tered by participants was } \\
\text { that they forgot to fill the } \\
\text { checking sheet. It was not } \\
\text { natural for them to make } \\
\text { the check }\end{array}$ & & & Part of the process & & \\
\hline
\end{tabular}




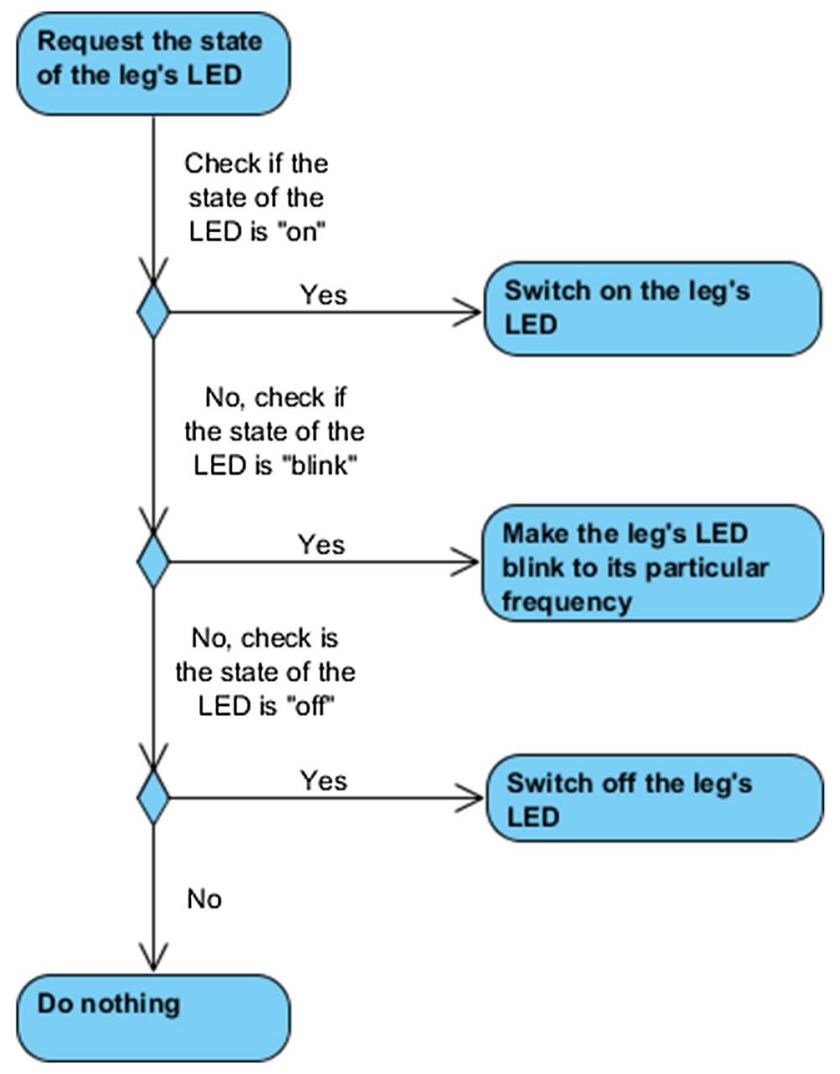

Fig. 11 Activity diagram for a LED
The participants were asked what would be the most important improvement to be made to the physical design of the workpieces and they answered that the embedded equipment should be smaller, lighter and integrated. In particular the point was made that the process was slower than it could be because of size and weight problems. A problem that has been encountered three times was that a sensor did not behave correctly; therefore it was suggested to implement a more reliable sensing method.

One participant noticed that instead of having the instruction "Move the part near the hole associated to the shape XXX" (where XXX is the name of the shape) it would have been more interesting to see the LED integrated on the sensor being switched on to indicate which was the corner where the leg must be assembled. The participant emphasised on the fact that it would be more visual and so, more intuitive.

It was also mentioned that the smart workpieces' capability would be very useful in a flexible manufacturing environment, particularly because the inspection process is automatically completed by the smart workpieces themselves. The summary of the above results is presented in Table 4 .

\subsection{Comparing manual versus smart assembly}

As seen Table 4, in terms of time, the manual scenario remains faster than the smart one $(2 \mathrm{~min}$ against $2 \mathrm{~min}$ and

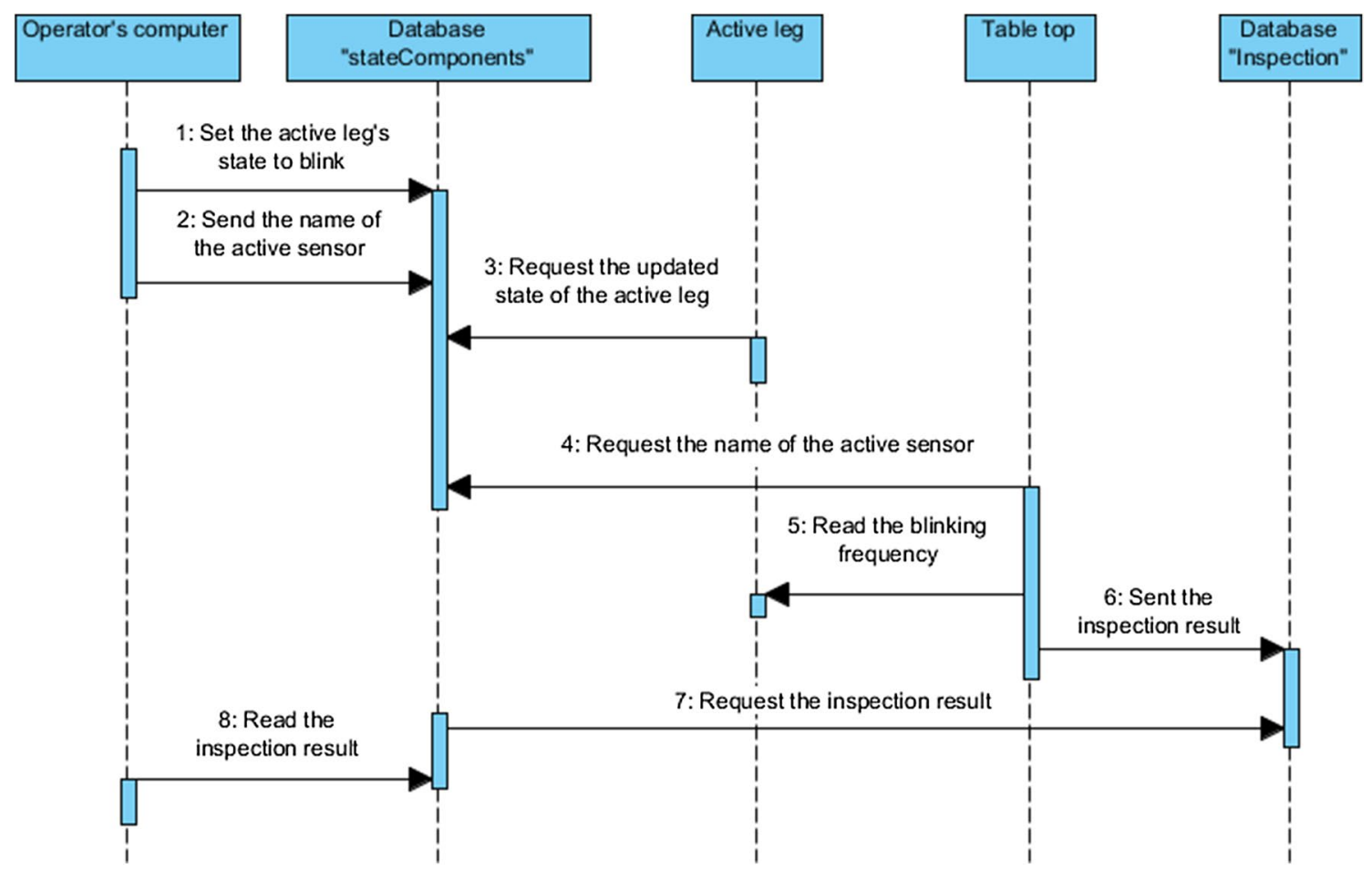

Fig. 12 Sequence diagram for smart inspect 
Table 3 Blinking frequency of the leg's LED

$\begin{array}{lll}\text { Leg number Associated to the corner } & \text { Blinking frequency } \\ 1 & 0.15 \mathrm{~s} \\ 2 & 0.20 \mathrm{~s} \\ 4 & 0.25 \mathrm{~s} \\ 4 & 0.30 \mathrm{~s}\end{array}$

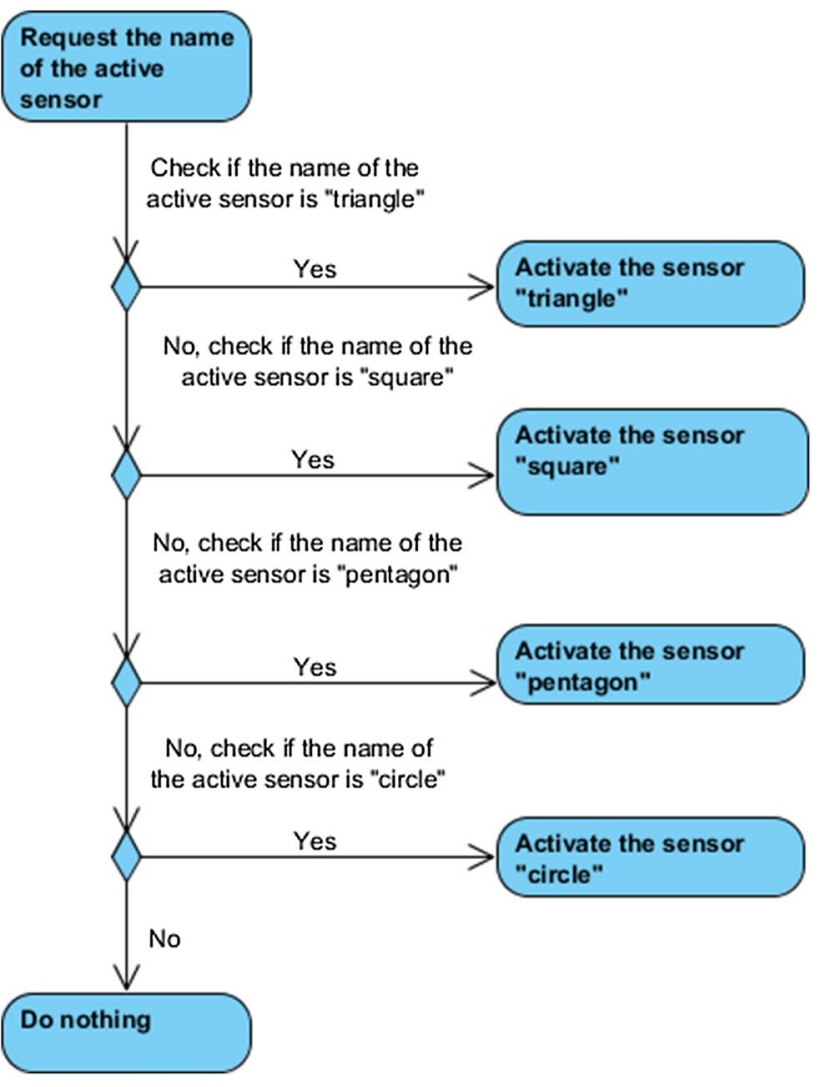

Fig. 13 Activity diagram for the colour sensors' activation

20-s). However, this can be explained by two factors. The first one is the fact that, because of their embedded equipment, the smart workpieces were heavier and so harder to manipulate. The second one is that the manual assembly is more natural as almost everybody has already built furniture by reading a manual. The novelty of the smart assembly was probably a disadvantage when it came to measuring the time the entire process took.

Nevertheless, as seen in Table 4, people using the manual assembly process sometimes forgot to complete necessary paper work. The smart assembly process offers the capability to digitize the assembly process so that the states of workpieces could be tracked, sources of assembly errors identified and reduced in the manufacturing line overall. As a result, the extra time spent using the smart assembly process could actually be saved eventually. Furthermore, it can be seen from Table 4, that people are willing to pay for such convenience in assembly and by extension digitization of the process.

\section{Discussion}

Although the table scenario remains a simple assembly scenario, it shows that there is a potential that workpieces can actually participate in an assembly operation by the use of active communication.

In order to make the workpieces smart, it was essential to identify what is a smart behaviour for a workpiece. However, workpieces have no real behaviour in the traditional assembly process. As a result, decomposition of a human manual process into its elemental motions using Therbligs was explored. The idea was to analyse, as much as possible, the behaviour of the human operator during a manual assembly process and then implement transferable motions into passive workpieces. In this work, the Therbligs (search/ select and find) that require information from the environment were chosen. These Therbligs capture how the human searches for an object/information, finds it and selects it for use. The idea in this paper was to transfer this informational element from the operator to the workpieces. We believe that doing so is the starting point to decentralize information and decision-making and facilitate a greater level of flexibility.

\subsection{Communication challenges}

Nevertheless, for the concept of smart workpieces to work effectively, each workpiece needs to know the status of the other workpieces in the assembly process as well as the progress of the overall assembly. As a result, the workpieces in this research had to continuously request access from the servers in order to receive status updates. On the scale of this use-case scenario, this was not a significant issue as only five workpieces were present. However, if there were more smart workpieces, it could have been very problematic. Indeed, if too many clients try to connect to a server at the same time, the server can become overloaded.

In order to solve this problem, protocols such as the MQ Telemetry Transport (MQTT) protocol could be used (OASIS, 2015). MQTT was designed as an extremely lightweight publish/subscribe messaging transport. This protocol is particularly used when it comes to connecting objects together in the Internet of Things philosophy. 


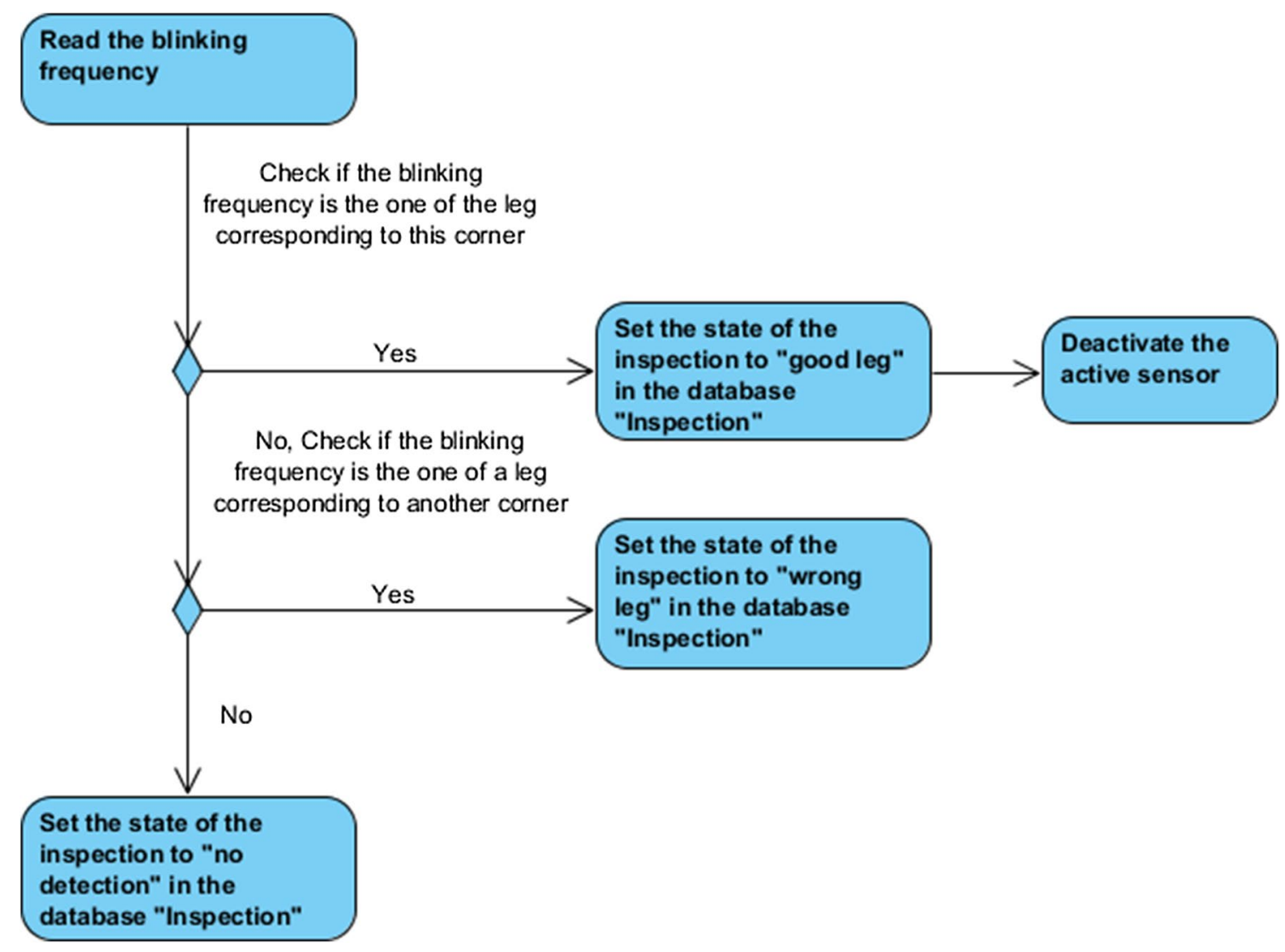

Fig. 14 Activity diagram for the active sensor

Table 4 Sequence of movement for manual and smart assemblies

Manual assembly Smart assembly

Read the instruction on the manual: "Please grasp the following leg

Read the instruction on the operator's screen: "Grasp the indicating leg (with picture of the leg below). Then, turn the page" and press 'Enter',

Grasp the leg

Grasp the leg

Turn the page

Press 'Enter'

Read the instruction on the manual: "Move the leg towards the corner associated to the shape (with picture of the shape below). Then, put

Read the instruction on the operator's screen: " "Move the part near the hole associated to the shape XXX" (where XXX is the name of the leg in the hole and screw until it is totally assembled. Fill the checking sheet" the shape). Then, put the leg's screw in the hole. Turn the leg until its LED is facing the sensor and then press 'Enter',

Take the checking sheet

Fill the sheet

Press 'Enter'

The inspection in the smart assembly is automatic. The sensor recognizes the blinking frequency of the leg. If the good leg is detected, the following message is read by the operator: "After inspection, it is confirmed that the leg is well associated to the corner XXX(where XXX is the name of the shape). Turn the leg until it is totally assembled and press 'Enter',',

Turn the leg until it is totally assembled

Go back to the manual. Then, read the instruction: "Turn the page"

Turn the page

The sequence is restarted for another leg

Press 'Enter'. The message "next leg”" appears on the screen and the sequence is restarted for another leg 


\subsection{Manufacturing challenges}

Furthermore, the use of smart workpieces could actual present more drawbacks. For example, it will make the manufacturing of workpieces more complex and might make assembling them more complex. In this research, the electronic components that made the workpieces smart had to be positioned very accurately on the workpieces to optimize the smart behaviour. In our case, when the sensors and the LED were not positioned perfectly, the sensor could not always detect the blinking frequency of the LED. If the LED is too close or too far from the sensor, the blinking frequency may not be detected. As a result, it increases the chances of having a defective workpieces because electronic faults might actually contribute to a defective part in addition to the traditional design flaws.

Another drawback is the fact that the components need to tolerate the manufacturing environment. Indeed, manufacturing environment can be very aggressive for such small pieces. For example, robots may apply a certain pressure on the workpieces that the components may not tolerate. Thus, there is a need for testing smart workpieces in manufacturing environment and this adds another layer of complexity into the manufacturing system.

Nevertheless, the use of smart components, and notably the use of an embedded microcomputer, also presents significant advantages. The first one is the fact that it makes the assembly process flexible and reconfigurable. This is due to the fact that the workpieces have their own intelligence. For example, in the use-case scenario, by changing two lines on the program located on the operator's microcomputer, it is possible to change the order of the assembly (the second leg can be assembled before the first one for example) and it is also possible to change the association leg-corner (a leg can be attributed to another corner and a corner can be associated to another leg).

As a consequence, the use of smart workpieces could become very useful when it comes to mass production of customised items. Another advantage of the smart components is that they can allow a continuous inspection of the assembled product, even during its utilisation. Equipped with the right sensors, the smart workpieces could inform the owner if their product is broken by, for example, informing that two constituent parts are not in contact anymore.

\subsection{Design and cost considerations}

The design of the workpieces is actually an important issue. It is difficult to imagine workpieces with too many external components as it will impact the design of the final product. Additionally, equipping workpieces with external components tends to make the workpieces heavier and harder to manipulate as was observed in the experiments. As a result, further investigation into how to integrate the components into the workpieces needs to be carried out. In order to integrate them, the way of manufacturing the workpieces would probably need to be reviewed because the assembly of the components into the workpieces needs to be integrated into the process. Perhaps sensor technologies could be integrated into the screws and bolts within furniture (with the LEDs being detachable either before purchase or after in the case of self-assembly leaving embedded sensors). Developing this new way of manufacturing the workpieces may be very costly. Furthermore, it requires precision technology in order to position the components in an optimized way as well as a need for people to program the smart behaviour of the smart workpieces efficiently. As a result, the cost of manufacturing increases due to smart workpieces thereby increasing the cost of the final product. However, in the survey for the test and validation, eight participants out of ten said they would agree to pay a bit more for their furniture to have this smart assembly guidance. Even though manufacturing smart workpieces seems more costly, it is important to notice that smart workpieces are a key to reach mass production of customised items and that could be a competitive advantage in the future.

\section{Conclusion}

Today's manufacturing facilities are facing the challenge of becoming fully flexible, adaptable, and reconfigurable in response to the demands of customised product production. The use of smart workpieces is seen as a potential solution to this need. This research has questioned the possibility for smart workpieces to participate actively in assembly operations. To do so, elemental assembly motions based on Therbligs were analysed and a use-case scenario was developed. This study then compared the manual assembly of an IKEA table to the assembly of the same table using smart workpieces.

Ten participants were invited to test the two assembly methods. The results showed that in its current form, the smart assembly was slower. Nevertheless, it reduces human errors and makes the assembly process more flexible, adaptable and reconfigurable. The possibility for smart workpieces to communicate in order to participate actively in assembly operation has been demonstrated, and further research in this direction is encouraged. In future work, the smart assembly must be tested in a manufacturing environment. Current manufacturing facilities have not been built with the aim to integrate smart components into workpieces and as a result, may require modification.

Further research could also look into the use of smart workpieces to manipulate robots. This could create a new way of manufacturing in which the smart workpieces 
themselves will indicate to the robot how it must manipulate them. Achieving this would allow the manufacturing facilities to become more adaptable, flexible and reconfigurable to achieve the efficient mass production of customised items.

Acknowledgments This project was supported by the Royal Academy of Engineering under the Research Chairs and Senior Research Fellowships scheme. Professor Ashutosh Tiwari is Airbus/RAEng Research Chair in Digitisation for Manufacturing at the University of Sheffield. The authors would also like to acknowledge the Manufacturing Technology Centre for their support.

Open Access This article is distributed under the terms of the Creative Commons Attribution 4.0 International License (http://creativeco mmons.org/licenses/by/4.0/), which permits unrestricted use, distribution, and reproduction in any medium, provided you give appropriate credit to the original author(s) and the source, provide a link to the Creative Commons license, and indicate if changes were made.

\section{References}

Alavudeen A, Venkateshwaran N (2008) Computer integrated manufacturing. PHI Learning Pvt. Ltd., New Delhi

Esmaeilian B, Behdad S, Wang B (2016) The evolution and future of manufacturing: a review. J Manuf Syst 39:79-100

Ferguson D (2000) Therbligs : The Keys to Simplifying Work. The Gilbreth Network [online]. http://gilbrethnetwork.tripod.com/ therbligs.html; 2000. Accessed 05/04/2019.

German Federal Government (2016) The new High-Tech Strategy Innovations for Germany [online]. Available: https://www.bmbf.de/ pub/HTS_Broschuere_eng.pdf. Accessed 05/05/2019

Gibbs S (2016) Mercedes-Benz swaps robots for people on its assembly lines, The Guardian Website [online]. https://www.thegu ardian.com/technology/2016/feb/26/mercedes-benz-robots-peopl e-assembly-lines. Accessed on 05/04/2019

Goldhar JD (1984) The Organizational Impacts of Computer Based Manufacturing. In Robotics and Artificial Intelligence. Springer, Berlin

Koestler A (1968) The ghost in the machine, Psychiatric. Communications 10(2):45-48

Lee J, Bagheri B, Kao HAA (2015) Cyber-Physical Systems architecture for Industry 4.0-based manufacturing systems. Manuf Lett 3:18-23

OASIS (2015) MQTT Standard [online]. http://docs.oasis-open.org/ $\mathrm{mqtt} / \mathrm{mqtt} / \mathrm{v} 3.1 .1 / \mathrm{mqtt}-\mathrm{v} 3.1 .1 . \mathrm{html} ; 2015$. Accessed 05/04/2019

Prabhu V, Tiwari A, Hutabarat W, Turner C (2015) Capture, digitisation and segmentation of human-workpiece interactions in a manual assembly operation using KinectTM. IJDE 6(1):61-85
Qu T, Zhang L, Huang Z, Dai Q, Chen X, Huang GQ, Luo H (2013) RFID-enabled smart assembly workshop management system. 2013 10th IEEE International Conference on Networking, Sensing and Control, ICNSC 2013:895-900

Rajkumar R, Lee I, Sha L, Stankovic J (2010) Cyber-physical systems. In: Proceedings of the 47th design automation conference on-DAC'10, 731

Savage CM (1987) Fifth Generation Management for Fifth Generation Technology (A Round Table Discussion). Society of Manufacturing Engineers, Dearborn, Michigan 1-30

Shrensker W (1990) CIM: a working definition. Society of Manufacturing Engineers Dearborn, Michigan, SME Blue Book Series

Suda H (1989) Future factory system formulated in Japan. Techno 22(10):15-25

Tao F, Zhang L, Venkatesh VC, Luo Y, Cheng Y (2011) Cloud manufacturing: a computing and service-oriented manufacturing model. Proc Inst Mech Eng 225:1969-1976

TAOS 2012 (2016) TCS3472 Color Light-to-Digital Converter with IR Filter Datasheet [online]. https://cdn-shop.adafruit.com/datas heets/TCS34725.pdf. Accessed 05/04/2019

Tsamis N, Giannikas V, McFarlane D, Lu W, Strachan J (2015) Adaptive storage location assignment for warehouses using intelligent products. Stud Comput Intell 594:271-279

Valckenaers P, Bonneville, F, Van Brussel, H, Bongaerts L, Wyns J (1994) Results of the holonic control system benchmark at KU Leuven. In: Proceedings of the Fourth International Conference on Computer Integrated Manufacturing and Automation Technology $128-133$

Velandia DMS, Kaur N, Whittow WG, Conway PP, West AA (2016) Towards industrial internet of things: crankshaft monitoring, traceability and tracking using RFID. Robot Comput Integ Manuf 41:66-77

Wang L, Haghighi A (2016) Combined strength of holons, agents and function blocks in cyber-physical systems. J Manuf Syst 40:25-34

Wang L, Törngren WM, Onori M (2015) Current status and advancement of cyber-physical systems in manufacturing. J Manuf Syst 37(2):517-527

Wu D, Greer MJ, Rosen DW, Schaefer D (2013) Cloud manufacturing: strategic vision and state-of-the-art. J Manuf Syst 32(4):564-579

Yu C, Xu X, Lu Y (2015) Computer-integrated manufacturing, cyberphysical systems and cloud manufacturing-concepts and relationships. Manuf Lett 6:5-9

Zhong RY, Dai QY, Qu T, Hu GJ, Huang GQ (2013) RFID-enabled real-time manufacturing execution system for mass-customization production. Robot Comput Integ Manuf 29(2):283-292

Publisher's Note Springer Nature remains neutral with regard to jurisdictional claims in published maps and institutional affiliations. 